\title{
Programmed cell death protein-1 inhibitors in the treatment of digestive system tumors in Chinese population: an observational study of effectiveness and safety
}

\author{
Wei Yang, Linlin Chang, Qianqian Guo, Jinhua Chen, Weijiang Yu, Wenzhou Zhang \\ Department of Pharmacy, Affiliated Cancer Hospital of Zhengzhou University, Henan Cancer Hospital, Zhengzhou, China \\ Contributions: (I) Conception and design: W Yang; (II) Administrative support: W Zhang; (III) Provision of study materials or patients: W Yu; (IV) \\ Collection and assembly of data: Q Guo, J Chen; (V) Data analysis and interpretation: L Chang; (VI) Manuscript writing: All authors; (VII) Final \\ approval of manuscript: All authors. \\ Correspondence to: Wenzhou Zhang. Department of Pharmacy, Affiliated Cancer Hospital of Zhengzhou University, Henan Cancer Hospital, 127 \\ Dongming Road, Zhengzhou 450003, China. Email: hnzzzwz@126.com.
}

Background Tumor of the digestive system is a common malignancy with high morbidity and mortality. Although programmed cell death-1 (PD-1) inhibitors have become an effective treatment strategies for many kinds of tumors, there is still some uncertainty in digestive tumors, including: (I) therapeutic effects of PD-1 inhibitors are relatively limited; (II) responses of digestive system tumors to immunotherapies are highly heterogeneous. In the present study, we investigated the outcomes of PD-1 inhibitors for digestive system tumors in Chinese patients to analyze factors that may affect the effects of immunotherapies in digestive system tumors.

Methods: Data were obtained from the Hospital Information System (HIS) of the Department of Digestive Oncology (Henan Cancer Hospital) between January 2019 and December 2019. Inclusion criteria included patients receiving the same PD-1 inhibitor continuously for advanced or recurrent/metastatic digestive system tumors. Indicators including age, sex, clinical diagnosis, height, weight, gene status, PD-1 inhibitors, treatment regimen, medication cycle, efficacy evaluation results, and adverse reactions were analyzed retrospectively. The clinical outcomes were progression-free survival (PFS) and safety.

Results: A total of 2,767 patients were discharged from HIS, of which 64 (37 male/27 female) were included in this study. Thirty-eight $(59.4 \%)$ of the patients were aged $<60$ years. Tumors included esophageal, gastric, liver, colorectal, and pancreatic cancer. Up until 30 June 2020, 51 patients were followed up to median progression-free survival (PFS), which was 5 months; the longest PFS was 18.5 months. There was no statistical significance in grouping according to sex, age and body mass index. Nevertheless, the median PFS differed statistically between monotherapy ( 9.4 months) versus combined therapy ( 4.7 months), and Cox regression analysis suggested that patients might benefit more from monotherapy than combined therapy. The incidence of adverse reactions was $47.7 \%$, with thyroid dysfunction the most common adverse reaction. The incidence of grade 3-4 adverse reactions was $9.2 \%$ and mainly included pulmonary infection, immuneassociated hepatitis, and severe oral ulcers.

Conclusions: In digestive tumors, especially for second-line treatment and beyond, PD-1 monotherapy might be more beneficial than combined therapy. However, this might be related to the patient's tolerance. Large-sample prospective studies are needed for confirmation.

Keywords: Immune checkpoint inhibitor (ICI); digestive system tumors; PD-1 monotherapy; adverse reactions

Submitted Jun 16, 2021. Accepted for publication Aug 11, 2021.

doi: $10.21037 /$ apm-21-1827

View this article at: https://dx.doi.org/10.21037/apm-21-1827 


\section{Introduction}

Immunotherapy is now an integral part of tumor treatment. In particular, the application of immune checkpoint inhibitors (ICIs) has marked a milestone in tumor therapy. Immune checkpoints are inhibitory signaling pathways in the immune system which suppress autoimmunity when activated, thereby preventing autoimmune disease but also favoring immune evasion by tumor cells. At present, ICIs primarily include monoclonal antibodies against cytotoxic $\mathrm{T}$ lymphocyte-associated antigen 4 (CTLA-4), programmed cell death-1 (PD-1) inhibitors, and programmed cell death ligand 1 (PD-L1) inhibitors (1). ICIs bypass tumor immune-evasion mechanisms and reestablish the immune system's antitumor response $(2,3)$. Clinical trials have demonstrated the efficacy of ICIs and the US Food and Drug Administration (FDA) has approved PD-1, PD-L1, and CTLA-4 inhibitors for the treatment of malignant tumors, including melanoma, renal carcinoma, and nonsmall cell lung cancer (NSCLC) $(4,5)$.

PD-1 is a transmembrane protein expressed on the surface of activated $\mathrm{T}$ cells (3). PD-L1 is a ligand for PD-1 and is normally expressed on antigen-presenting cells. Activation of PD-1 signaling by PD-L1 results in a downregulated $T$ cell response through reduced cytokine production and a subsequent dampening of the inflammatory response. Tumor cells capitalize on this signaling pathway to evade the immune system. In melanomas and other tumors, overexpression of PD-L1 and its subsequent over-activation of $\mathrm{PD}-1$ signaling prevents $\mathrm{T}$ cell responses, allowing the tumors to avoid the anti-tumor immune response (6). PD-1/PD-L1 inhibitors work by blocking the binding of PD-L1 to PD-1, enabling the antitumor immune response to occur (2-4).

Digestive system tumors are characterized by high incidence and relatively poor overall prognosis (7). Some tumors, including those of the liver, stomach, colorectal, and pancreas, are already in an advanced stage at the time of diagnosis, limiting the efficacies of chemotherapy and radiotherapy. The National Comprehensive Cancer Network's (NCCN) Clinical Practice Guidelines for Gastric Cancer (Version 2) issued in 2020 recommended the use of PD-1 inhibitor pembrolizumab for second-line treatment and subsequent lines of treatment of advanced microsatellite instability-high or deficient mismatch repair gastric cancer, as well as third-line and subsequent lines of treatment of PD-L1 positive gastric adenocarcinomas (8). Digestive system tumors are highly heterogeneous. Colorectal cancer of different subtypes, for example, exhibits variable responses to identical immunotherapeutic regimens varied significantly among patients with different subtypes. Most notably, PD-1 inhibitors are not effective for pMMR-MSI-L colorectal cancer (9). Various phase II and III clinical trials for ICIs in digestive-system tumor treatment have been completed or are currently ongoing $(10,11)$. Nonetheless, many patients with digestive system tumors have been treated with ICIs, mostly as second- or third-line treatments. Also, the timing of immunotherapies, combinations with other antitumor regimens and biomarkers for efficacy have not been adequately studied.

The objective of this study was to analyze factors that may affect the effects of immunotherapies in digestive system tumors by observing the efficacy and safety of PD-1 inhibitors in patients with digestive system tumors.

We present the following article in accordance with the STROBE reporting checklist (available at https://dx.doi. org/10.21037/apm-21-1827).

\section{Methods}

\section{Patients}

Data from patients with digestive system tumors who were hospitalized and treated in the Department of Digestive Oncology, Henan Cancer Hospital, Henan, China, between January 2019 and December 2019 were collected from the Hospital Information System (HIS). Henan Cancer Hospital is the only tertiary oncology hospital in Henan Province. The hospital currently has 2,991 ward beds, with approximately 600,000 outpatient visits and 170,000 hospitalized patients annually. The patient inclusion criteria were as follows: (I) diagnosed with digestive system tumors, including esophageal cancer, gastric cancer, colorectal cancer, primary liver cancer, pancreatic cancer, and gallbladder cancer; (II) the presence of advanced tumors, or postoperatively metastatic or recurrent tumors; and (III) treated with PD-1 inhibitors. The exclusion criteria were as follows: (I) currently participating in a clinical trial of PD-1 inhibitors; (II) incomplete data; or (III) received PD-1 inhibitors for neo-adjuvant therapy. Follow-up information was obtained from medical record, and PFS was determined based on the evaluation of clinical response by attending physicians (Figure 1). The study was conducted in accordance with the Declaration of Helsinki (as revised in 2013). The study was approved by the Ethics Committee of the Henan Cancer Hospital. Informed consent was waived 


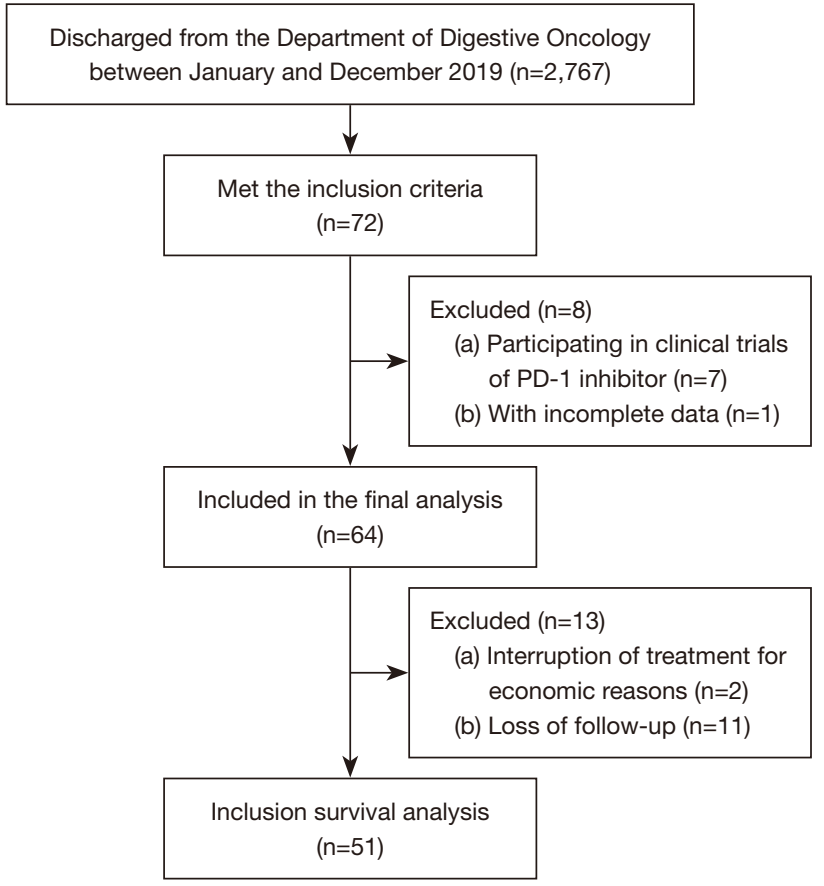

Figure 1 Flow diagram of the inclusion of patients.

due to the retrospective study design.

\section{Indicators and definitions}

The following data were collected for patients included in the study: sex, age, clinical diagnosis, body mass index (BMI), PD-1 inhibitor type, treatment regimen and dose, drug combination, medication cycle, progression-free survival (PFS), genetic status, and adverse reactions.

BMI was calculated according to the equation: $\mathrm{BMI}=$ body weight $(\mathrm{kg}) /$ height $\left(\mathrm{m}^{2}\right)$. Overweight and obesity were diagnosed according to the Guidelines for the Prevention and Management of Overweight and Obesity in Chinese Adults issued by the Ministry of Health of China. In brief, a BMI $<20 \mathrm{~kg} / \mathrm{m}^{2}$ was considered underweight, a BMI of $20-24.9 \mathrm{~kg} / \mathrm{m}^{2}$ was considered normal weight, and a BMI $\geq 25.0 \mathrm{~kg} / \mathrm{m}^{2}$ was considered obese $(12,13)$.

PFS was defined as the time from initiation of PD-1 inhibitor treatment to disease progression. Disease progression was defined according to the Response Evaluation Criteria in Solid Tumors (RECIST Version 1.1).

\section{Genotyping for microsatellite instability (MSI)}

Genotyping for MSI was undertaken with reverse transcription polymerase chain reaction (RT-PCR) and DNA sequencing by the Targeted Gene Measurement Center of Henan Cancer Hospital, using the Mx3000P QPCR System (Agilent, Santa Clara, CA, USA) and ABI3130 genetic analyzer (Thermo Fisher Scientific, Waltham, MA, USA). Patients were genotyped as microsatellite stable (MSS), microsatellite instability-low (MSI-L), and microsatellite instability-high (MSI-H).

Adverse reactions recorded in the HIS were classified according to the National Cancer Institute's Common Terminology Criteria for Adverse Events (NCI-CTCAE 4.0).

For the known confounding factors, the inclusion criteria of the research objects are limited at the time of design, and the confounding bias is controlled by certain statistical processing methods in the data analysis stage, such as hierarchical analysis, the use of regression models and so on.

\section{Statistical analysis}

R version 3.5.2 software (http://www.R-project.org/) was used for statistical analysis and result visualization. Normal distribution tests were performed for all the continuous variables. Data with normal distribution were described using mean and standard deviation (SD), while nonnormally distributed data were described using median and range. Categorical data were described with frequencies and percentages.

Kaplan-Meier (K-M) analysis was used to visualize survival curves. Independent factors influencing PFS were identified by single- and multivariate Cox regression models. Multivariate Cox regression was performed using the stepwise method. Subgroup analysis of PFS was performed using the log-rank test. $\mathrm{P}<0.05$ was considered statistically significant.

\section{Results}

\section{Patient characteristics}

Between January and December 2019, 2,767 patients were discharged from the Department of Digestive Oncology at the Henan Cancer Hospital. Among these patients, 72 met the initial inclusion criteria. However, 7 patients were excluded for having participated in clinical trials of PD-1 inhibitors and 1 was excluded for incomplete data, consequently, 64 patients were included in the analysis. Of these patients, $37(57.8 \%)$ were male, 27 female, and $38(59.4 \%)$ were aged $<60$ years; two patients $(3.1 \%)$ were 
Table 1 Baseline demographic characteristics of the patients

\begin{tabular}{|c|c|}
\hline Variables & Number (\%) \\
\hline \multicolumn{2}{|l|}{ Sex } \\
\hline Male & $37(57.8)$ \\
\hline Female & $27(42.2)$ \\
\hline \multicolumn{2}{|l|}{ Age group (years) } \\
\hline$<60$ & $38(59.4)$ \\
\hline $60-69$ & $15(23.4)$ \\
\hline $70-79$ & $9(14.1)$ \\
\hline$\geq 80$ & $2(3.1)$ \\
\hline \multicolumn{2}{|l|}{ Primary disease } \\
\hline Esophageal cancer & $11(17.2)$ \\
\hline Gastric cancer & $9(14.1)$ \\
\hline Colorectal cancer & $11(17.2)$ \\
\hline Intestinal cancer & $2(3.1)$ \\
\hline Tumor of biliary system & $12(18.8)$ \\
\hline Liver cancer & $13(20.3)$ \\
\hline $\begin{array}{l}\text { Cancer of middle-to-lower esophageal } \\
\text { segment- cardia cancer }\end{array}$ & $1(1.6)$ \\
\hline $\begin{array}{l}\text { Squamous carcinoma of esophagogastric } \\
\text { junction }\end{array}$ & $1(1.6)$ \\
\hline Pancreatic cancer & $4(6.3)$ \\
\hline \multicolumn{2}{|l|}{ Body mass index $\left(\mathrm{kg} / \mathrm{m}^{2}\right)$} \\
\hline$<20$ & 20 (31.3) \\
\hline $20-24.9$ & $31(48.4)$ \\
\hline$\geq 25$ & $13(20.3)$ \\
\hline \multicolumn{2}{|l|}{ Gene status } \\
\hline MSS & $14(21.9)$ \\
\hline MSI-L & $3(4.7)$ \\
\hline MSI-H & $8(12.5)$ \\
\hline Missing data & $39(60.9)$ \\
\hline
\end{tabular}

MSS, microsatellite stable; MSI-L, microsatellite instability-low; $\mathrm{MSI}-\mathrm{H}$, microsatellite instability-high.

$\geq 80$ years old. Twenty (31.3\%), 31 (48.4\%), and 13 (20.3\%) of the patients had a BMI $<20,20-24.9$, and $\geq 25 \mathrm{~kg} / \mathrm{m}^{2}$, respectively. The patients were diagnosed with esophageal cancer, gastric cancer, colorectal cancer, intestinal cancer, gallbladder cancer, liver cancer, and pancreatic cancer, respectively. Genotyping results for microsatellite instability
Table 2 Treatments of the patients with the anti-PD- 1 antibodies

\begin{tabular}{lc}
\hline PD-1 inhibitor & Number (\%) \\
\hline Agent & $13(20.3)$ \\
Pembrolizumab & $5(7.8)$ \\
Nivolumab & $19(29.7)$ \\
Sintilimab & $11(17.2)$ \\
Toripalimab & $14(21.9)$ \\
Camrelizumab & $1(1.6)$ \\
Camrelizumab/sintilimab & $1(1.6)$ \\
Unknown & \\
Treatment regimen & $20(31.3)$ \\
Monotherapy & $16(25.0)$ \\
Combined therapy & $24(37.5)$ \\
Combined with anti-angiogenetic agents & $4(6.2)$ \\
Combined with chemotherapeutic and & \\
anti-angiogenetic agents & $25(39.1)$ \\
\hline Lines of treatment & $20(31.2)$ \\
First line & $19(29.7)$ \\
\hline &
\end{tabular}

showed that 39 patients $(60.9 \%)$ did not undergo genotyping or had unavailable test results. Among the 25 patients for whom data was available, there were 14,3 , and 8 with MSS, MSI-L, and MSI-H genes, respectively (Table 1).

\section{Types of PD-1 inbibitors and medication regimen}

The PD-1 inhibitors used to treat patients included pembrolizumab, nivolumab, sintilimab, toripalimab, and camrelizumab. The medication regimens included monotherapy, combined therapy, combined therapy with anti-angiogenetic agents, and combined therapy with chemotherapeutic and anti-angiogenetic agents. PD-1 inhibitors were used for the first-, second-, third-line and beyond treatments in $20(31.2 \%), 19(29.7 \%)$, and 25 (39.1\%) patients, respectively (Table 2).

\section{Clinical efficacy}

Eleven patients did not readmit to hospital for further treatment and thus PFS could not be accurately evaluated for 


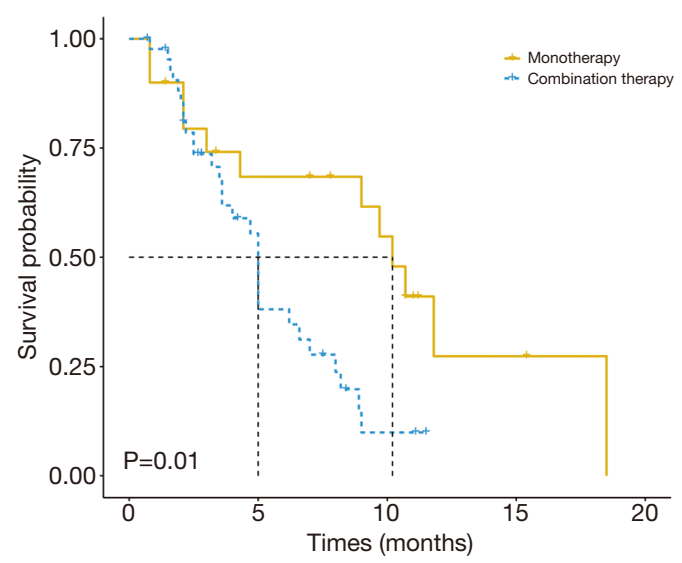

Figure 2 Progression-free survival of patients.

these patients. A further 2 patients, whose medication cycles were 4 and 6 cycles, respectively, discontinued their drug therapy for financial reasons. As a result, 13 of the 64 patients in this study could not be followed up.

Data for the 51 patients who were followed to the median PFS were collected up to June 30, 2020 and included in the Cox regression analysis (Figure 2). At that time, 10 patients were still on medication and had not reached PFS. Four patients discontinued treatment due to intolerable toxic side effects, including infection, thrombocytopenia, and hypoglycemia. The median PFS was 5 months and the longest PFS was 18.5 months. Cox regression also showed that combined therapy was an independent factor influencing PFS of the patients (Table 3).

\section{ICI-induced adverse reactions}

ICI therapy involved symptomatic treatment and supplementary treatment with glucocorticoids. Four patients discontinued PD-1 inhibitor treatment due to intolerable toxic effects. According to the patients' medical records and related laboratory examination results, the incidence of adverse drug reaction was $47.7 \%$. The most common adverse reaction was thyroid dysfunction, followed by liver dysfunction, particularly in patients with liver cancer or cholangiocarcinoma for whom the increase of alanine aminotransferase was aggravated and bilirubin level was abnormal after drug application. The incidence of grades 3-4 adverse reactions was $9.2 \%$ and mainly included pulmonary infection, immune-associated hepatitis, and severe oral ulcers (Figure 3).

\section{Discussion}

This study evaluated real-world data from patients in Henan Province, China who were treated with PD-1 inhibitors for digestive system tumors. The findings indicated a greater survival rate in patients treated with PD-1 monotherapy compared with those treated with combined therapies. Additionally, the results showed that mild to moderate adverse drug reactions occurred in almost $50 \%$ of patients, although the toxicity profile was manageable. Real-world data of this nature is useful for supporting clinical decisionmaking in the many patient populations where complete clinical efficacy and safety data have yet to emerge. Both the European Medicines Agency (EMA) and the FDA encourage the analysis of real-world data for drug safety and efficacy monitoring $(14,15)$. The results of this study also suggest that analyzing patients' MSI-H status could help to individualize treatment.

PD-1 plays a pivotal role in normal immunity, acting as a checkpoint protein. It is primarily found on the surface of antigen-experienced $\mathrm{T}$ cells and was first shown to be a regulator of immune responses in PD-1-deficient mouse models (16). Activation of PD-1 signaling downregulates T cell-mediated responses and thereby limits tissue damage in normal immune system homeostasis (3-6). In many tumors, the overexpression of PD-L1 and its subsequent overactivation of PD-1 signaling prevents $\mathrm{T}$ cell responses from enabling the tumors to avoid anti-tumor immune responses (6). PD-1/PD-L1 blockade has been a major advance in cancer treatment.

Currently, PD-1 inhibitors are mainly approved for the treatment of malignant melanoma, lung cancer, and renal cancer (1). For patients with such tumors, sufficient evidence exists for the clinical application of PD-1 inhibitors. Evidence for PD-1 inhibitors in digestive-system tumor treatment remains inconclusive. For example, earlier clinical trials in colorectal cancer patients (17-19) demonstrated that only some patients benefited from immunotherapies. The findings showed that ICIs only exerted substantial beneficial effects in patients with colorectal cancer and deficient mismatch repair (dMMR) but did not induce effective immune responses in patients with mismatch repair proficient (pMMR) colorectal cancer. This study mainly included patients with digestive system tumors, of which liver cancer accounted for the highest proportion $(\mathrm{n}=19)$, followed by esophageal cancer $(n=14)$. Preliminary results from the combined immunotherapy plus targeted treatment for liver cancer patients showed promising results. The 
Table 3 Multivariate Cox analysis of PFS

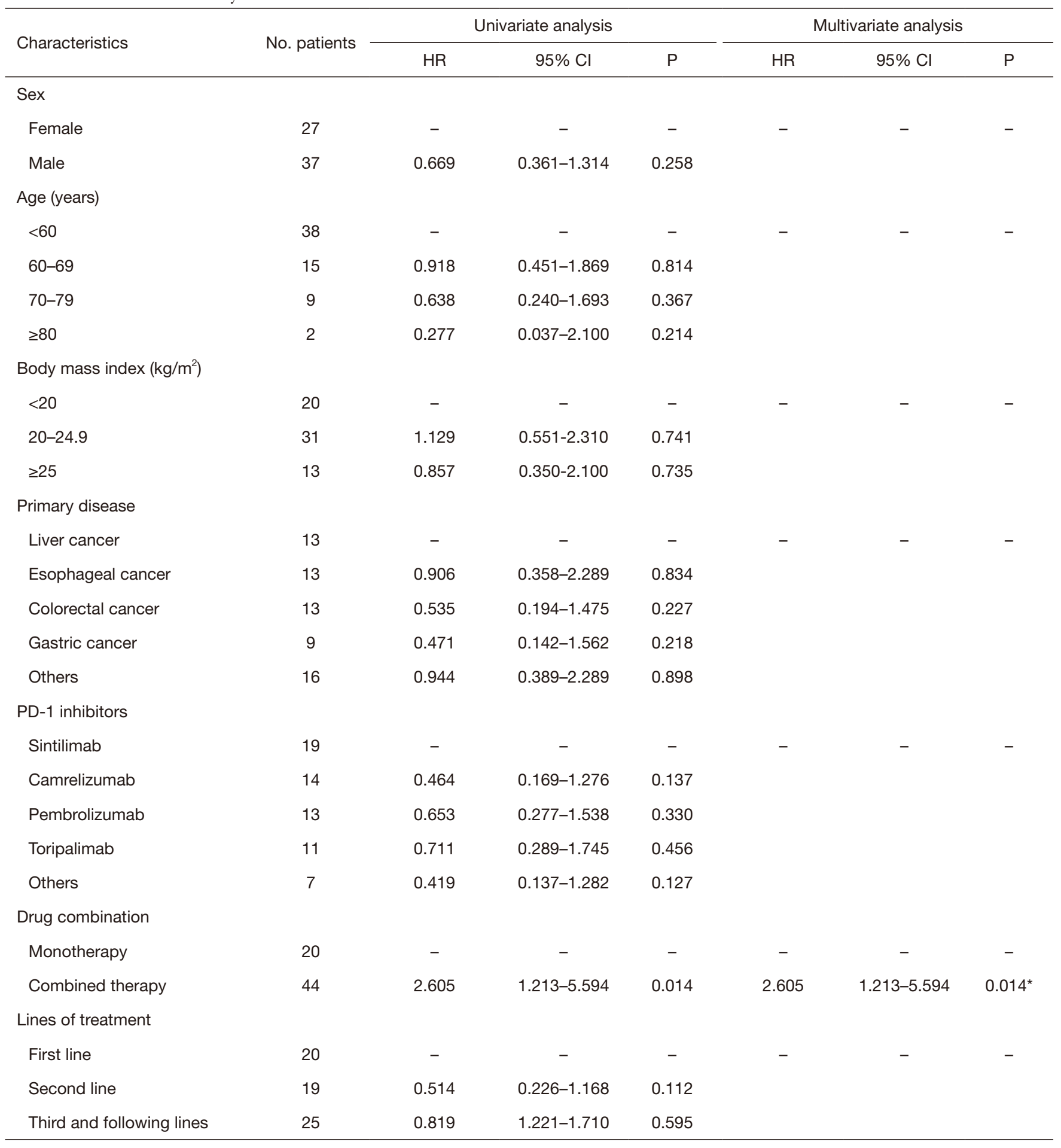

Combined therapy was the independent factor influencing the PFS of the patients ( $\left.{ }^{*} \mathrm{P}=0.0144\right)$. PFS, progression-free survival. 


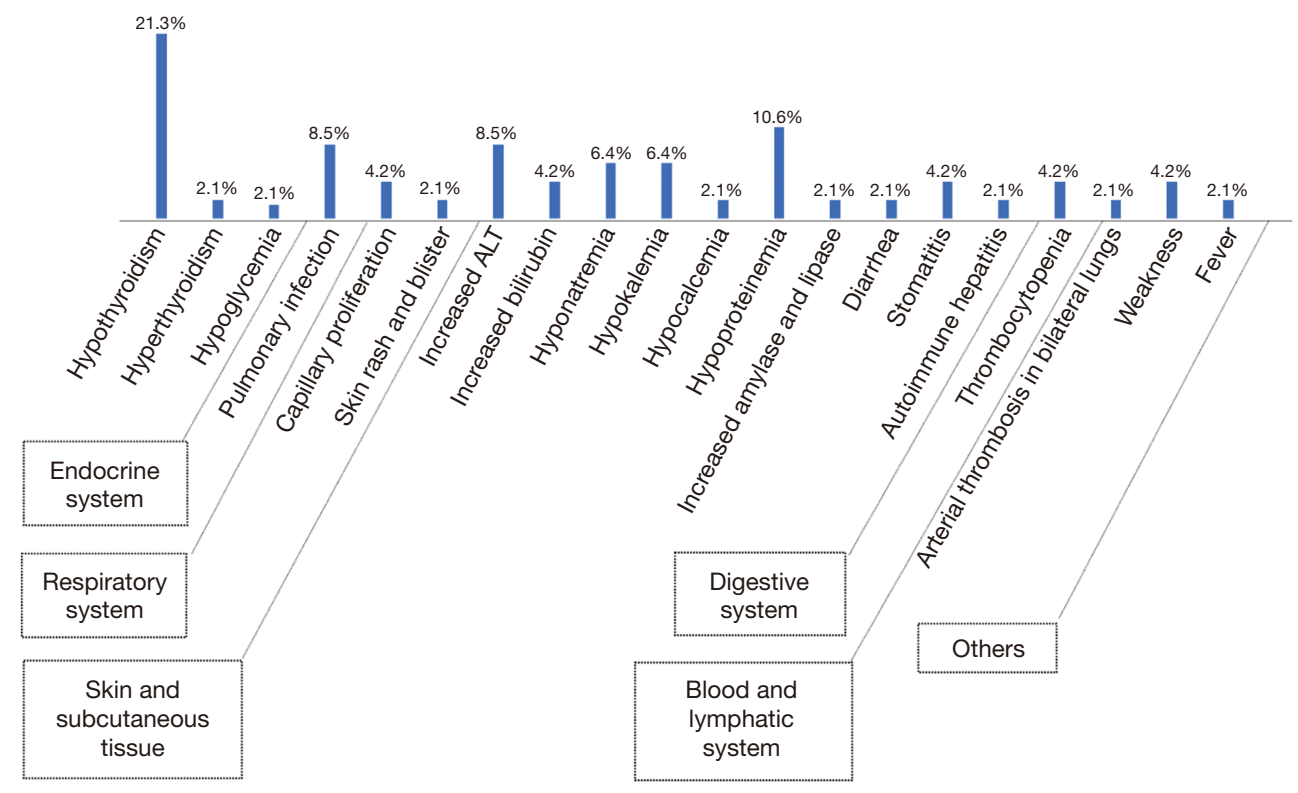

Figure 3 Clinical manifestations and incidence of ICI-induced adverse reactions. ICI, immune checkpoint inhibitor; ALT, alanine aminotransferase.

KEYNOTE-024 study, which investigated the safety and efficacy of lenvatinib + pembrolizumab in treating advanced liver cancer patients, showed that the median PFS was 9.7 months, and 6- and 12 -month survival was $83.3 \%$ and $59.8 \%$, respectively (20). In this study, $46.7 \%$ of the patients received ICIs for the first-line therapy of primary liver cancer. In addition, 1 patient received monotherapy with nivolumab for the second-line treatment, which involved 16 medication cycles. However, medication cycles for most liver cancer patients were $<4$ cycles, especially for patients who also had severe ascites. Therefore, patient screening based on such predictors is necessary and could help to identify which patients might benefit most from ICI treatment, thereby increasing efficacy.

In this study, more male patients than female were included. This sex distribution is supported by epidemiological data that shows that the prevalence of digestive system disorders is higher in males (8). In addition, most patients $(58.5 \%)$ were $<60$ years of age. Lichtenstein et al. (21) investigated the effects of age on the survival of patients with NSCLC who received immunotherapy and showed that the survival benefits varied by age group. For instance, patients aged 60-69 years had a higher probability of PFS and overall survival (OS). However, the adverse reactions related to immunotherapy did not significantly vary among the different age groups.
Previous studies have demonstrated an association between BMI and tumor development. For instance, being underweight (BMI $<18.5 \mathrm{~kg} / \mathrm{m}^{2}$ ) increases the risks of gastric and liver cancers in male patients, while obesity (BMI $\geq 28.0 \mathrm{~kg} / \mathrm{m}^{2}$ ) increases the risks of colorectal cancer in males, ovarian cancer in female patients, and breast cancer in postmenopausal women $(22,23)$. High BMI was also shown to be an independent positive predictor for the survival of patients after surgical treatments for early NSCLC, combined chemotherapy with paclitaxel and carboplatin for advanced diseases, and radiotherapy for bone metastases. Another study in patients with NSCLC showed that BMI and OS were linearly correlated; patients with a high BMI benefited more from ICI treatments and therefore baseline $\mathrm{BMI}$ could be an independent stratification factor for future ICI treatment (13). In this study, $20.0 \%$ of patients had a BMI of $\geq 25.0 \mathrm{~kg} / \mathrm{m}^{2}$, but the sample size was too small to observe a significant relationship between BMI and PD-1 efficacy.

Previous studies have suggested that $\mathrm{dMMR} / \mathrm{MSI}-\mathrm{H}$ tumors have hyper-mutations which are manifested in large concentrations of frameshift peptides. As novel antigens, frameshift peptides could induce the immune response of lymphocytes that have infiltrated into tumors. Several studies have already demonstrated that dMMR/ MSI-H colorectal and liver cancer patients have relatively 
high responses to ICI treatment $(24,25)$. There were 8 patients with MSI-H in this study. The median number of medication cycles for this group was 7, which was higher than the 4.5 cycles for patients overall in this study. Of the 8 patients with MSI-H, 7 were followed up to PFS. The median PFS for this group was 11.2 months, which was higher than the overall PFS of 5 months.

With the wide application of ICIs in tumor treatments, immunotherapy-related adverse events (irAEs) have attracted considerable attention. Previous studies have shown that the incidence of irAEs is about $60-80 \%$. However, the incidence of irAEs varies according to different ICIs. In addition, the incidence of irAEs is also higher in patients who received combined immunotherapy than monotherapy $(26,27)$. In this study, the irAE incidence for the 21 patients who were treated with ICI monotherapy was $42.8 \%$, with mainly mild to moderate clinical manifestation of thyroid dysfunction. However, for patients who received combined therapy, including PD-1 inhibitors combined with cytotoxic agents and/or anti-angiogenetic agents, the irAE incidence was $45.4 \%$, suggesting an influence on one or more organ systems. In this group, manifestations included pulmonary infection, immune-associated hepatitis, thrombocytopenia, and severe hyponatremia/hypokalemia. Although the understanding of irAEs has advanced somewhat, most irAEs are managed by discontinuation of ICI treatment or through the use of glucocorticoids. Due to the lagging effects and persistency of immune responses, some irAEs may appear relatively late or even several months after ICI discontinuation. Therefore, the identification, monitoring, and follow up of irAEs should be undertaken throughout the duration of ICI treatment.

Several previous studies have shown that combined therapy with PD-1 inhibitor and anti-angiogenetic agents results in better outcomes. In this study, PD-1 inhibitors combined with anti-angiogenetic agents, as well as PD-1 inhibitors combined with chemotherapeutic agents, were used. However, the survival analysis suggests that patients might benefitted more from monotherapy with a PD-1 inhibitor than from the combined therapies. The reasons for this could be as follows: (I) $65 \%$ of the censored patients were in the combined therapy group and the proportion of patients not included in follow-up was higher in this group; (II) COX regression in the current study was based on only 64 patients, this study was a retrospective analysis with a relatively small sample size and unmeasured confounders may have influenced the relationship; and (III) due to the extent of adverse reactions, the patients may have had better tolerance to the PD-1 inhibitor monotherapy. More importantly, many of the confounding factors that could affect PFS are not known. Also, as a retrospective analysis, the results of the analyses are subject to patient selection bias.

The limitations of this study include the relatively small sample size that limits the extent of statistical inference, the diversity of tumor treatments received by the patients, and incomplete genotyping of the sample for microsatellite instability markers. In addition, unknown lifestyle, physiological, or other clinical variables may have influenced the findings.

\section{Conclusions}

Progress has been achieved in treating digestive system tumors with ICIs. Currently, several phase II/III clinical trials are ongoing, and more studies with subjects from various hospitals are needed to improve these findings and increase the evidence base for the use of ICIs in cancers of the digestive system.

\section{Acknowledgments}

Funding: This work was supported by the joint construction project of Henan Provincial Medical Science and Technology Tack Key Project (LHGJ20190648).

\section{Footnote}

Reporting Checklist: The authors have completed the STROBE reporting checklist. Available at https://dx.doi. org/10.21037/apm-21-1827

Data Sharing Statement: Available at https://dx.doi. org/10.21037/apm-21-1827

Conflicts of Interest: All authors have completed the ICMJE uniform disclosure form (available at https://dx.doi. org/10.21037/apm-21-1827). The authors have no conflicts of interest to declare.

Ethical Statement: The authors are accountable for all aspects of the work and ensuring that questions related to the accuracy or integrity of any part of the work are appropriately investigated and resolved. The study was conducted in accordance with the Declaration of Helsinki (as revised in 2013). The study was approved by the Ethics 
Committee of the Henan Cancer Hospital. Informed consent was waived due to the retrospective study design. Open Access Statement: This is an Open Access article distributed in accordance with the Creative Commons Attribution-NonCommercial-NoDerivs 4.0 International License (CC BY-NC-ND 4.0), which permits the noncommercial replication and distribution of the article with the strict proviso that no changes or edits are made and the original work is properly cited (including links to both the formal publication through the relevant DOI and the license). See: https://creativecommons.org/licenses/by-nc-nd/4.0/.

\section{References}

1. Himmel ME, Saibil SD, Saltman AP. Immune checkpoint inhibitors in cancer immunotherapy. CMAJ 2020;192:E651.

2. Alsaab HO, Sau S, Alzhrani R, et al. PD-1 and PDL1 Checkpoint Signaling Inhibition for Cancer Immunotherapy: Mechanism, Combinations, and Clinical Outcome. Front Pharmacol 2017;8:561.

3. Akinleye A, Rasool Z. Immune checkpoint inhibitors of PD-L1 as cancer therapeutics. J Hematol Oncol 2019;12:92.

4. Michot JM, Bigenwald C, Champiat S, et al. Immunerelated adverse events with immune checkpoint blockade: a comprehensive review. Eur J Cancer 2016;54:139-48.

5. Pardoll DM. The blockade of immune checkpoints in cancer immunotherapy. Nat Rev Cancer 2012;12:252-64.

6. Zou W, Chen L. Inhibitory B7-family molecules in the tumour microenvironment. Nat Rev Immunol 2008;8:467-77.

7. Nagtegaal ID, Odze RD, Klimstra D, et al. The 2019 WHO classification of tumours of the digestive system. Histopathology 2020;76:182-8.

8. NCCN Clinical Practice Guidelines in Oncology (NCCN Guidelines). Gastric Cancer. Version 3.2020. Fort Washington: National Comprehensive Cancer Network; 2020.

9. Oliveira AF, Bretes L, Furtado I. Review of PD-1/PDL1 Inhibitors in Metastatic dMMR/MSI-H Colorectal Cancer. Front Oncol 2019;9:396.

10. Hirsch D, Gaiser T, Merx K, et al. Clinical responses to PD-1 inhibition and their molecular characterization in six patients with mismatch repair-deficient metastatic cancer of the digestive system. J Cancer Res Clin Oncol 2021;147:263-73.

11. Sun J, Zheng Y, Mamun M, et al. Research progress of PD-1/PD-L1 immunotherapy in gastrointestinal tumors.
Biomed Pharmacother 2020;129:110504.

12. WHO Expert Consultation. Appropriate body-mass index for Asian populations and its implications for policy and intervention strategies. Lancet 2004;363:157-63.

13. Kichenadasse G, Miners JO, Mangoni AA, et al. Association Between Body Mass Index and Overall Survival With Immune Checkpoint Inhibitor Therapy for Advanced Non-Small Cell Lung Cancer. JAMA Oncol 2020;6:512-8.

14. Skovlund E, Leufkens HGM, Smyth JF. The use of realworld data in cancer drug development. Eur J Cancer 2018;101:69-76.

15. Burock S, Meunier F, Lacombe D. How can innovative forms of clinical research contribute to deliver affordable cancer care in an evolving health care environment? Eur J Cancer 2013;49:2777-83.

16. Nishimura H, Nose M, Hiai H, et al. Development of lupus-like autoimmune diseases by disruption of the PD-1 gene encoding an ITIM motif-carrying immunoreceptor. Immunity 1999;11:141-51.

17. Overman MJ, McDermott R, Leach JL, et al. Nivolumab in patients with metastatic DNA mismatch repair-deficient or microsatellite instability-high colorectal cancer (CheckMate 142): an open-label, multicentre, phase 2 study. Lancet Oncol 2017;18:1182-91.

18. Le DT, Durham JN, Smith KN, et al. Mismatch repair deficiency predicts response of solid tumors to PD-1 blockade. Science 2017;357:409-13.

19. Le DT, Uram JN, Wang H, et al. PD-1 Blockade in Tumors with Mismatch-Repair Deficiency. N Engl J Med 2015;372:2509-20.

20. Reck M, Rodríguez-Abreu D, Robinson AG, et al. Updated Analysis of KEYNOTE-024: Pembrolizumab Versus Platinum-Based Chemotherapy for Advanced NonSmall-Cell Lung Cancer With PD-L1 Tumor Proportion Score of 50\% or Greater. J Clin Oncol 2019;37:537-46.

21. Lichtenstein MRL, Nipp RD, Muzikansky A, et al. Impact of Age on Outcomes with Immunotherapy in Patients with Non-Small Cell Lung Cancer. J Thorac Oncol 2019;14:547-52.

22. Friedenreich CM, Ryder-Burbidge C, McNeil J. Physical activity, obesity and sedentary behavior in cancer etiology: epidemiologic evidence and biologic mechanisms. Mol Oncol 2021;15:790-800.

23. Avgerinos KI, Spyrou N, Mantzoros CS, et al. Obesity and cancer risk: Emerging biological mechanisms and perspectives. Metabolism 2019;92:121-35.

24. Huyghe N, Baldin P, Van den Eynde M. Immunotherapy 
with immune checkpoint inhibitors in colorectal cancer: what is the future beyond deficient mismatch-repair tumours? Gastroenterol Rep (Oxf) 2020;8:11-24.

25. Kreidieh M, Mukherji D, Temraz S, et al. Expanding the Scope of Immunotherapy in Colorectal Cancer: Current Clinical Approaches and Future Directions. Biomed Res Int 2020;2020:9037217.

26. Gu Y, Zhang H, Liu Z, et al. Different patterns of treatment-related adverse events of programmed cell

Cite this article as: Yang W, Chang L, Guo Q, Chen J, Yu W, Zhang W. Programmed cell death protein-1 inhibitors in the treatment of digestive system tumors in Chinese population: an observational study of effectiveness and safety. Ann Palliat Med 2021;10(8):9015-9024. doi: 10.21037/apm-21-1827 death-1 and its ligand-1 inhibitors in different cancer types: A meta-analysis and systemic review of clinical trials. Asia Pac J Clin Oncol 2020;16:e160-78.

27. Sakakida T, Ishikawa T, Uchino J, et al. Safety and tolerability of PD-1/PD-L1 inhibitors in elderly and frail patients with advanced malignancies. Oncol Lett 2020;20:14.

(English Language Editor: A. Muijlwijk) 